\title{
Asynchronously Coupled Resonant Junctions for Diplexers and Multi-port Filtering Networks
}

\author{
Eugene A. Ogbodo, Yun Wu, Peter Callaghan, and Yi Wang* \\ Department of Engineering Science, University of Greenwich \\ Kent, ME4 4TB, United Kingdom \\ *Yi.Wangegre.ac.uk
}

\begin{abstract}
This paper proposes the use of asynchronously coupled resonator junctions in the design of diplexers. This is to ease the control and implementation of the external couplings at the common port of an all-resonator-based diplexer. A comparison study has been performed between a conventional diplexer and the proposed diplexer. To further explore the possibilities of the proposed junction technique, a four-port multi-port filtering networks was proposed and designed to function as a double diplexer. Good agreements have been achieved between the measurements of the prototype devices and the simulations.
\end{abstract}

\section{INTRODUCTION}

Diplexers are three-port devices used in the communication systems for transmitting and receiving signals through a shared antenna. A diplexer is made up of two channel filters connected by a junction. In the case of conventional diplexers, the junction is usually formed of transmission lines in the form of T-junction [1] or Y-junction [2]. In addition to these, hybrid couplers [3], manifolds [4] or circulators [5] were also used. Recently, a new design approach which utilises resonant junctions was introduced into diplexer designs [6]-[8]. Circuit miniaturisation is achieved when the resonating junction not only serves to split/combine signals but also as a resonant pole contributing to the frequency selectivity. Such techniques were reported in [8], [9] using single-mode junction resonators and in [10]-[12] using dual-mode junction resonators such as T-shaped resonators [13], steppedimpedance resonators (SIRs) [14] and patches [6]. The need for the junction resonator to couple in three ways to the common port as well as to the two channels complicates the implementation. Single-mode junction resonators restrict the overall bandwidth of the signal that is allowed in a diplexer. For dual-mode junctions, the main challenge is the control of the external couplings to the two respective modes from a common port.

This work is built on from the design concept of resonant junctions but proposes the use of asynchronously coupled resonator junctions to ease the control and implementation of the external couplings at the common port. Two diplexers based on a conventional $\mathrm{T}$-junction and the proposed resonant junction, as illustrated in Fig. 1(a) and (b), were first designed and compared. The proposed junction structure was then applied to demonstrate the realisation of a four-port filtering network as illustrated in Fig. 1(c).

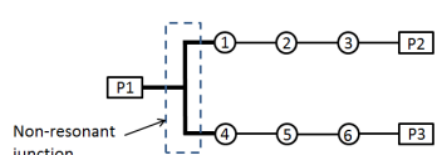

(a)

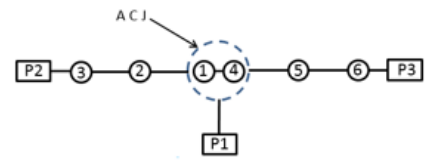

(b)

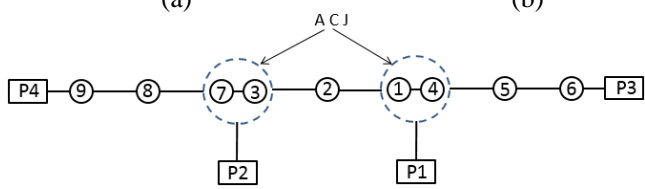

(c)

Fig. 1. Coupling topologies of (a) a conventional diplexer, (b) the proposed diplexer and (c) a 4-port filtering network, where ACJ stands for asynchronously coupled resonator junction.

\section{DIPLEXER DESIGN AND COMPARISON}

\section{A. Layouts of Diplexers}

Fig. 1(a) demonstrates the topology of a conventional Tjunction diplexer. The non-resonant $\mathrm{T}$-junction is designed using $\lambda / 4$ transmission lines. Fig. 1(b) demonstrates the proposed diplexer coupling topology where the dashed circle indicates the asynchronous coupled resonant junction (ACJ). The junction is essentially a pair of asynchronously tuned resonators of different resonating frequencies coupled together. The coupling topologies of Fig. 1(a) and (b) are translated into their circuit layouts using microstrip technology as presented in Fig. 2 and Fig. 3 respectively.

The conventional T-junction diplexer in Fig. 2 and the proposed asynchronous coupled resonant junction diplexer in Fig. 3 are both designed to operate at $1.8 \mathrm{GHz}$ and $2.1 \mathrm{GHz}$ with a $4 \%$ fractional bandwidth (FBW), and $20 \mathrm{~dB}$ return loss on each channel. Two channel filters operating at $1.8 \mathrm{GHz}$ and $2.1 \mathrm{GHz}$ were first separately designed to specifications using hairpin resonators. To join the channel filters, the T-junction transmission line was used for the conventional diplexer as shown in Fig. 2. In the case of the proposed coupled resonant junction diplexer, the first resonators of both channel filters were asynchronously coupled together. A single feed-line from the common port was sequentially coupled to these two resonators through edge coupling. The technique resulted in a compact circuit. More importantly the external couplings from the common port to the two resonators serving the two respective channels can be readily controlled. 


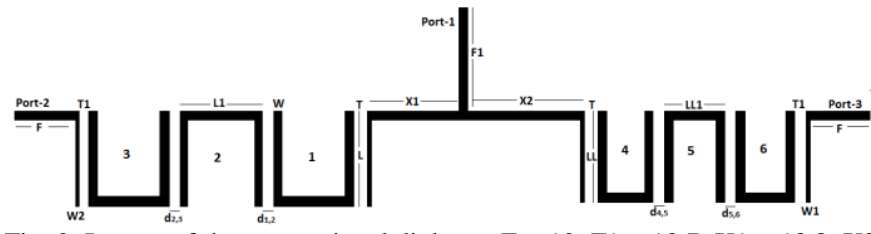

Fig. 2. Layout of the conventional diplexer. $\mathrm{F}=10, \mathrm{~F} 1=13.7, \mathrm{X} 1=13.8, \mathrm{X} 2$ $=17.6, \mathrm{~T}=0.3, \mathrm{~T} 1=0.4, \mathrm{~W}=1.2, \mathrm{~W} 1=0.6, \mathrm{~W} 2=0.6, \mathrm{~L}=11.5, \mathrm{~L} 1=9.95$, $\mathrm{LL}=11, \mathrm{LL} 1=6.59, \mathrm{~d}_{1,2}=1.77, \mathrm{~d}_{2,3}=1.77, \mathrm{~d}_{4,5}=1.75, \mathrm{~d}_{5,6}=1.75$. Unit: $\mathrm{mm}$.

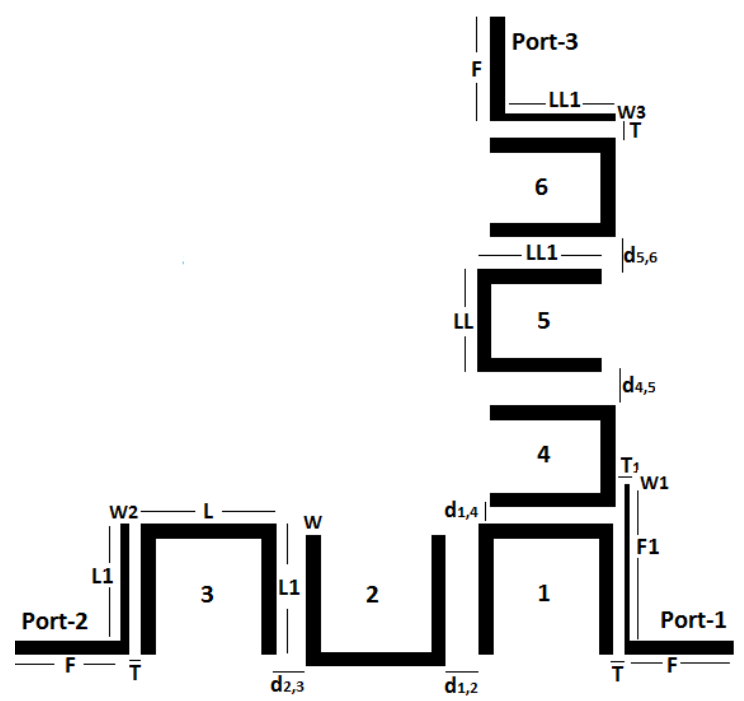

Fig. 3. Proposed diplexer. $\mathrm{F}=10, \mathrm{~T}=0.4, \mathrm{~T} 1=0.2, \mathrm{~W}=1.2, \mathrm{~W} 1=0.4$, $\mathrm{W} 2=$ $0.6, \mathrm{~W} 3=0.6, \mathrm{~L}=9.6, \mathrm{~L} 1=11.5, \mathrm{LL}=6.6, \mathrm{LL} 1=11, \mathrm{~d}_{1,2}=1.7, \mathrm{~d}_{1,4}=0.2$, $\mathrm{d}_{2,3}=1.7, \mathrm{~d}_{4,5}=1.65, \mathrm{~d}_{5,6}=1.7$. Unit: $\mathrm{mm}$.

\section{B. Asynchronous Coupling and $Q_{\text {ext }}$}

The diplexers in Fig. 3 are made up of two filters of $\mathrm{f} 1=$ $1.8 \mathrm{GHz}$ and $\mathrm{f} 2=2.1 \mathrm{GHz}$ with $4 \% \mathrm{FBW}$ each. These filters are designed to meet the three-pole Chebyshev ripple factor of $0.043 \mathrm{~dB}$ with a lowpass prototype derived from [15]-[16] with g-values of $\mathrm{g} 0=\mathrm{g} 4=1.0, \mathrm{~g} 1=\mathrm{g} 3=0.8516$, and $\mathrm{g} 2=$ 1.1032. The g-values are used in obtaining the coupling coefficients and external Q-factors used for the physical dimensioning of the resonators. Using the general filter synthesis method, the coupling coefficients and external quality factors were derived from (1) and (2).

$$
\begin{gathered}
M_{1,2}=M_{2,3}=M_{4,5}=M_{5,6}=\frac{F B W}{\sqrt{g_{1} g_{2}}}=0.041 \\
Q_{e x 1(L)}=Q_{e x 1(H)}=Q_{e x 2}=Q_{e x 3}=\frac{g_{1} g_{0}}{F B W}=21.29
\end{gathered}
$$

where Qex1(L) and Qex1(H) are the external Q-factors from the common port to the low and high channel respectively. After individually designing the channel filters to specification, the first resonators of the $1.8 \mathrm{GHz}$ and $2.1 \mathrm{GHz}$ filters were coupled together to define the two channel frequencies as shown in Fig. 4.

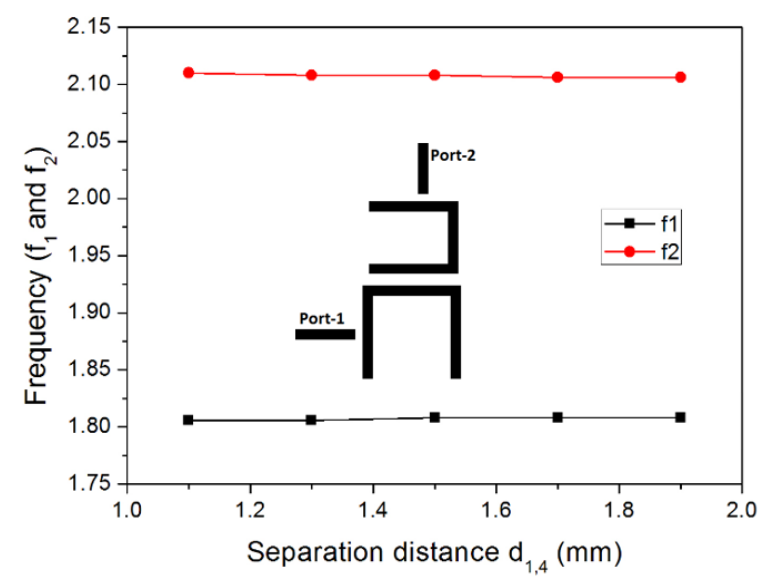

Fig. 4. Asynchronous coupling between resonator 1 and 4 .

To achieve the external quality factors $\left(\mathrm{Q}_{\mathrm{ext}}\right)$ from the common port to the asynchronous coupled resonator junction, a coupled feed line was used as shown in Fig. 5. Port-2 and port-3 in Fig. 5 were weakly coupled to the resonators whereas the coupling gap, width and length of the feed line were adjusted to achieve the required $\mathrm{Q}_{\mathrm{ext}}$ using (3).

$$
Q_{e x 1(L) /(H)}=\frac{f_{1(L) /(H)}}{\Delta f_{1(L) /(H)}}
$$

where $f_{1(L) /(H)}$ and $\Delta f_{1(L) /(H)}$ are the centre frequencies and the $3 \mathrm{~dB}$ bandwidths of the resonance curves corresponding to the low and high channel. Fig. 5(a) presents a graph of the typical responses. Fig. 5(b) shows the simulated current distribution at the resonator junction at $1.8 \mathrm{GHz}$ and $2.1 \mathrm{GHz}$. It is evident that the ACJ acts as a signal splitter.
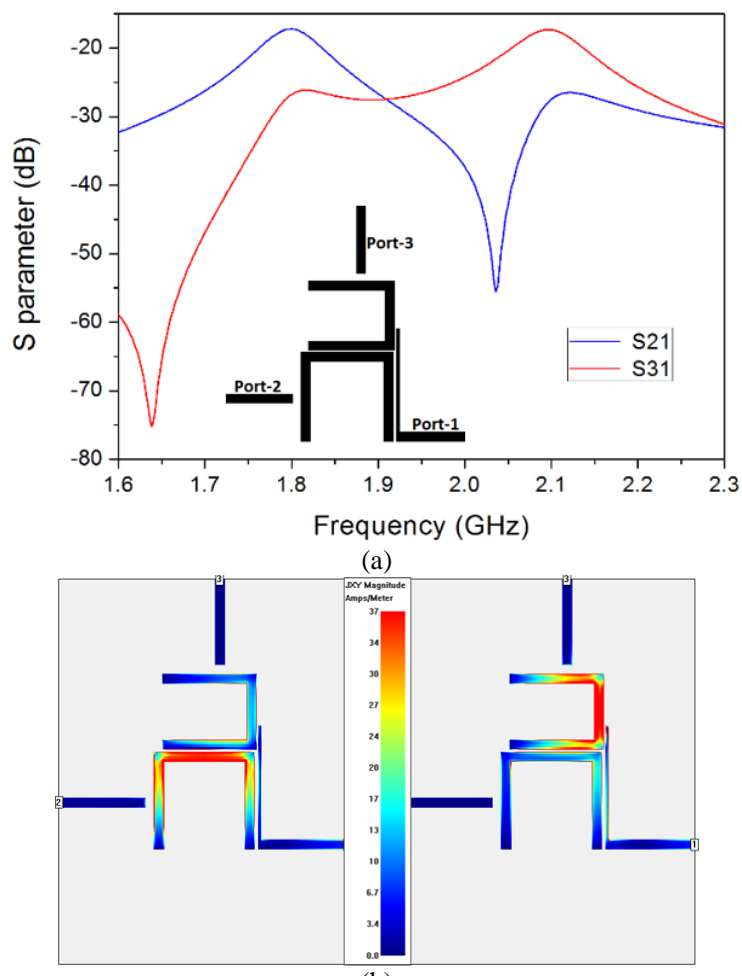

(b)

Fig. 5. (a) Simulated responses of the configuration used to extract $Q_{\text {ext }}$; (b) Simulated current distribution at $1.8 \mathrm{GHz}$ (left) and $2.1 \mathrm{GHz}$ (right). 


\section{Performance Comparison}

The EM software Sonnet is used for the simulation. Fig. 2 and 3 present the achieved physical dimensions after optimization. Fig. 6 compares the achieved simulated responses of the proposed diplexer (solid lines) with the conventional $\mathrm{T}$-junction diplexer (dash lines). While the two diplexers achieved similar rejection in the high band, the resonator junction provided less rejection in the low band. This is expected due to the lack of isolation between the two coupled resonators which is one shortcoming of resonant junctions. Rogers 3010 substrates with a thickness of $1.27 \mathrm{~mm}$, a relative permittivity of 10.8 and a loss tangent of 0.0022 were used. Measurements were taken using Agilent Network Analyser N5230A. The measured responses of the two diplexers are presented in Fig. 7. Good agreement between the simulations and measurements has been achieved.

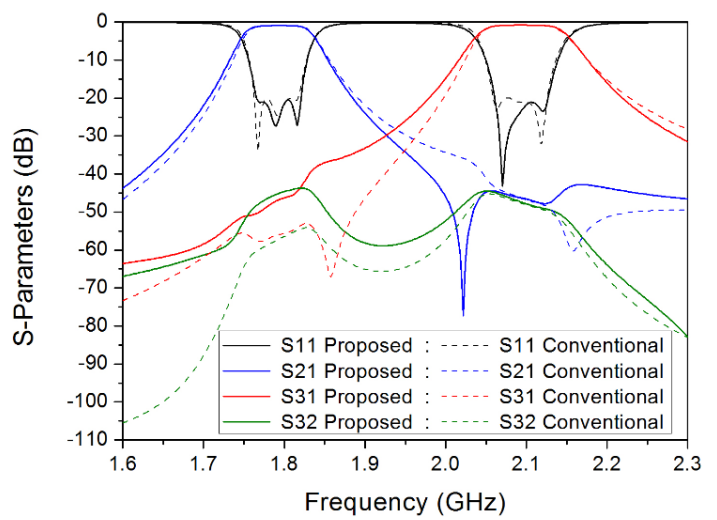

Fig. 6. Simulated responses of the proposed and the conventional diplexers.

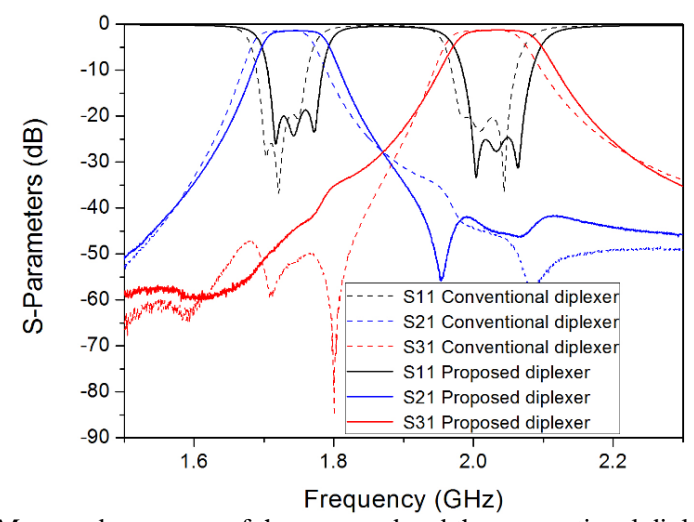

Fig. 7. Measured responses of the proposed and the conventional diplexer.

\section{4-PORT FILTERING NETWORK}

To further explore the potentials of the coupled resonator junctions, a four-port filtering network in the form of two back-to-back diplexers is designed as illustrated in Fig. 1(c). It is not targeting any specific application at this stage but to demonstrate the capability of realizing multi-port coupledresonator networks. From the coupling topology, the network contains two asynchronous coupled resonator junctions, three filters (three bands) and four ports. Fig. 8 presents the design layout where resonators 1 and 4 as well as resonators 3 and 7 represent the asynchronous coupled resonator junctions. The resonators 1-3 forms the channel of $1.8 \mathrm{GHz}$, resonators 4-6 and resonators 7-9 forms the channels of $2.1 \mathrm{GHz}$ and 2.6 $\mathrm{GHz}$ respectively.

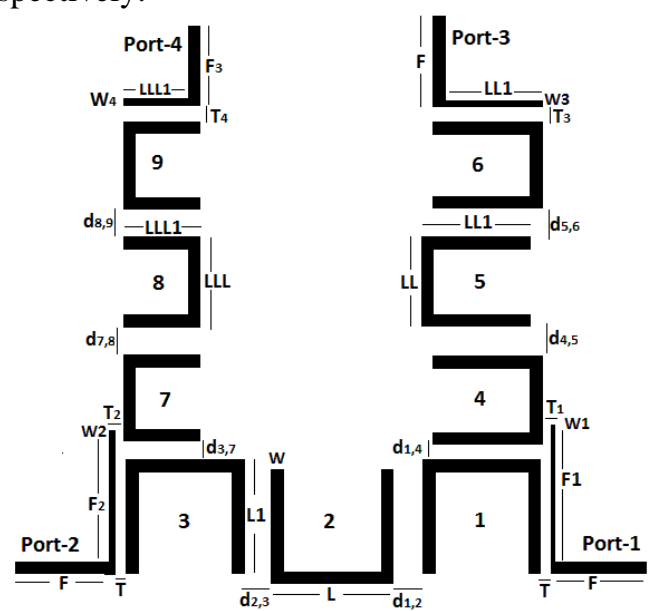

Fig. 8. Layout of the proposed double diplexer. $\mathrm{F}=10, \mathrm{~F} 1=15, \mathrm{~F} 2=15, \mathrm{~T}=$ $0.4, \mathrm{~T} 1=0.2, \mathrm{~T} 2=0.38, \mathrm{~W}=1.2, \mathrm{~W} 1=0.4, \mathrm{~W} 2=0.6, \mathrm{~W} 3=0.6, \mathrm{~L}=9.6, \mathrm{~L} 1$ $=11.5, \mathrm{LL}=6.6, \mathrm{LL} 1=11, \mathrm{~d}_{1,2}=1.46, \mathrm{~d}_{1,4}=0.2, \mathrm{~d}_{2,3}=1.48, \mathrm{~d}_{3,7}=0.22, \mathrm{~d}_{4,5}=$ $1.65, \mathrm{~d}_{5,6}=1.7, \mathrm{~d}_{7,8}=1.84, \mathrm{~d}_{8,9}=1.82$. Unit: $\mathrm{mm}$.

The design in Fig. 8 is built on from the diplexer in Fig. 3 by adding on a $2.6 \mathrm{GHz}$ filter using another asynchronous coupled resonator junction. That is resonator 7 coupled to resonator 3. The coupling topology of this circuit offers this design an opportunity to function as switchable diplexers or even multi-input and multi-output filtering networks. Fig. 9 presents two possible choices of selected diplexer channels. Fig. 9(a) shows the frequency duplexing at $1.8 \mathrm{GHz}$ and 2.1 $\mathrm{GHz}$ when the common port is Port-1. Fig. 9(b) shows the duplexing of $1.8 \mathrm{GHz}$ and $2.6 \mathrm{GHz}$ when the common port is Port-2. All the return losses are below $20 \mathrm{~dB}$. Measurements and simulations show reasonably good agreement except for the frequency shift of $10 \mathrm{MHz}$. This is believed to due to the thinning of the substrate from milling as well as the tolerance of the dielectric constant of this high permittivity anisotropic substrate material. Fig. 10 presents the isolation response between port- 1 and port-4, port- 2 and port-3 as well as port-3 and port- 4 . The solid lines represent the measured responses while the dashed lines represent the simulated responses.

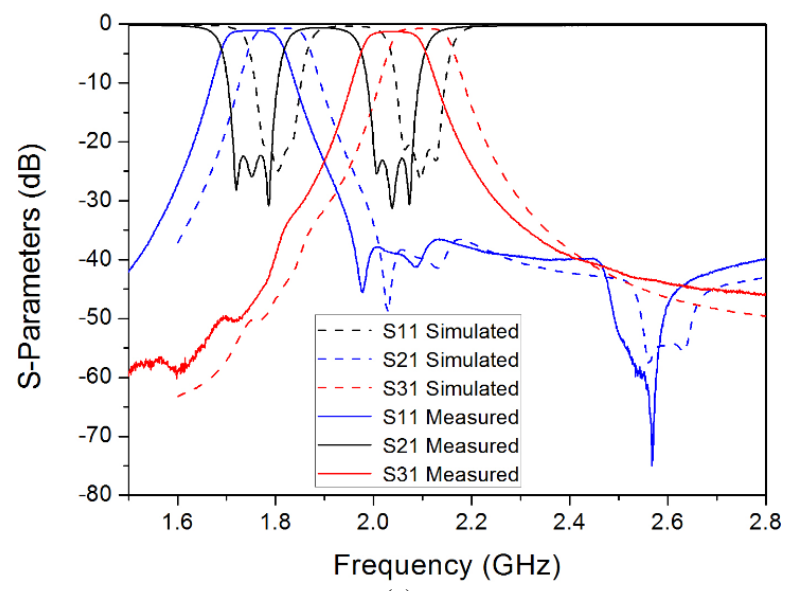

(a) 


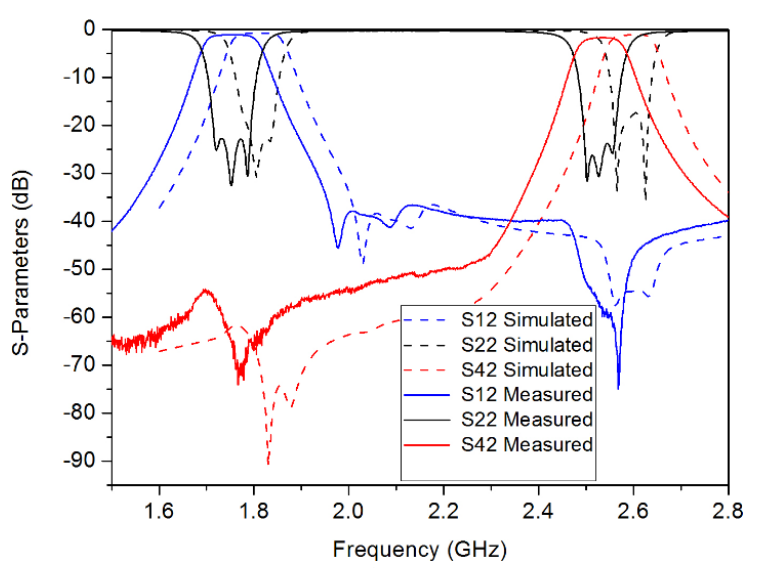

(b)

Fig. 9. Simulated and measured duplexing responses of the design in Fig. 8 when (a) Port-1 is the common port and (b) Port-2 is the common port.

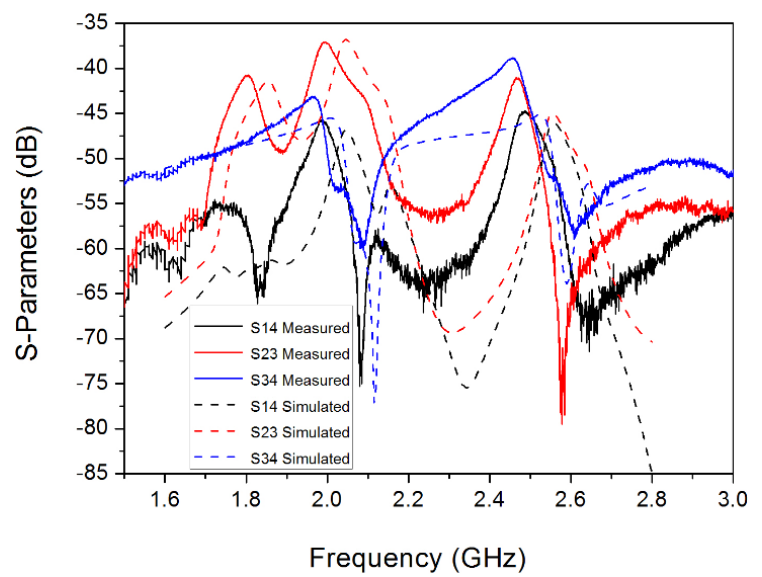

Fig. 10. Simulated and measured isolation responses of $S_{14}, S_{23}$ and $S_{34}$ of the design in Fig. 8.

\section{CONCLUSIONS}

In this work, asynchronously coupled resonant junctions are proposed and investigated for applications in resonatorbased diplexers and multi-port filtering networks. The proposed resonant junction structure succeeded in easing the implementation of external couplings at the common ports as compared with previous reported single-mode or dual-mode junctions. The comparison between a conventional $\mathrm{T}$-junction diplexer and the proposed resonant junction diplexer has concluded a more compact structure of the proposed diplexer but at the cost of channel rejection. Better isolation between the asynchronously coupled resonators is desired at the junction. It is also worth mentioning that the asynchronous coupled resonator junction technique can be used in designing diplexers or multiplexers with both narrowly or widely separated channels. To further demonstrate the capability of the coupled junction, a four-port filtering network in the form of a double diplexer was presented. Depending on the choice of the common port, different duplexing schemes can be realized. Although this four-port device is not currently conceived for any specific application, the underlining concept of a generic multi-port resonator network could be explored for circuit functions such as switchable diplexers or multi-input and multi-output filtering networks.

\section{REFERENCES}

[1] T. Yang, P. L. Chi and T. Itoh, "High Isolation and Compact Diplexer Using the Hybrid Resonators," in IEEE Microwave and Wireless Components Letters, vol. 20, no. 10, pp. 551-553, Oct. 2010.

[2] K. L. Wu and W. Meng, "A Direct Synthesis Approach for Microwave Filters With a Complex Load and Its Application to Direct Diplexer Design," in IEEE Transactions on Microwave Theory and Techniques, vol. 55, no. 5, pp. 1010-1017, May 2007.

[3] Y. L. Cheng, H. W. Chen, P. D. Huang and C. Y. Chang, "A W-band quadrature hybrid coupled substrate integrated waveguide diplexer," 2015 Asia-Pacific Microwave Conference (APMC), Nanjing, 2015, pp. $1-3$.

[4] T. Skaik and M. Lancaster, "Coupled Resonator Diplexer without External Junctions," Journal of Electromagnetic Analysis and Applications, Vol. 3 No. 6, 2011, pp. 238-241.

[5] C. E. Saavedra, "Diplexer using a circulator and interchangeable filters," 2008 7th International Caribbean Conference on Devices, Circuits and Systems, Cancun, 2008, pp. 1-5.

[6] E. A. Ogbodo, Y. Wu and Y. Wang, "Microstrip diplexers with dualmode patch resonant junctions," 2016 46th European Microwave Conference (EuMC), London, United Kingdom, 2016, pp. 1155-1158.

[7] X. Guan et al., "A novel microstrip diplexer with a common square ring resonator for WCDMA," 2016 IEEE International Workshop on Electromagnetics: Applications and Student Innovation Competition (iWEM), Nanjing, 2016, pp. 1-3.

[8] Y. Wu, Y. Wang and E. A. Ogbodo, "Microstrip wideband diplexer with narrow guard band based on all-resonator structures," 2016 46th European Microwave Conference (EuMC), London, United Kingdom, 2016, pp. 1163-1166.

[9] G. Macchiarella, "Synthesis of Star-Junction Multiplexers," in IEEE Microwave Magazine, vol. 12, no. 6, pp. 101-109, Oct. 2011.

[10] T. F. Skaik and D. A. Tubail, "Novel multiplexer topologies based on coupled resonator structures," Microwave Symposium (MMS), 2015 IEEE 15th Mediterranean, Lecce, 2015, pp. 1-4.

[11] X. Shang, Y. Wang, X. Wenlin, M. J. Lancaster, "Novel Multiplexer Topologies Based on All-Resonator Structures," IEEE Trans. Microwave Theory and Techniques, vol 61, no. 11, 2013, pp. 3838 3845

[12] M. L. Chuang and M. T. Wu, "Microstrip diplexer design ussing common T-shaped resonator," IEEE Microwave. Wireless Components. Letter., vol. 21, no. 11, pp. 583-585, Nov. 2011.

[13] X. Guan, F. Yang, H. Liu and L. Zhu, "Compact and High-Isolation Diplexer Using Dual-Mode Stub-Loaded Resonators," in IEEE Microwave and Wireless Components Letters, vol. 24, no. 6, pp. 385 387, June 2014

[14] C. F. Chen, T. Y. Huang, C. P. Chou and R. B. Wu, "Microstrip Diplexers With Common Resonator Sections for Compact Size, But High Isolation," IEEE Transactions on Microwave Theory and Techniques, Vol. 54, No. 5, May 2006.

[15] J.-S. Hong "Microstrip Filters for RF/Microwave Applications," John Wiley \& Sons, Inc. 2011.

[16] D. M. Pozar, Microwave Engineering. 3rd ed. New York: Wiley, 2005. 